\title{
Existence, Uniqueness, and Comparative Statics in Contests
}

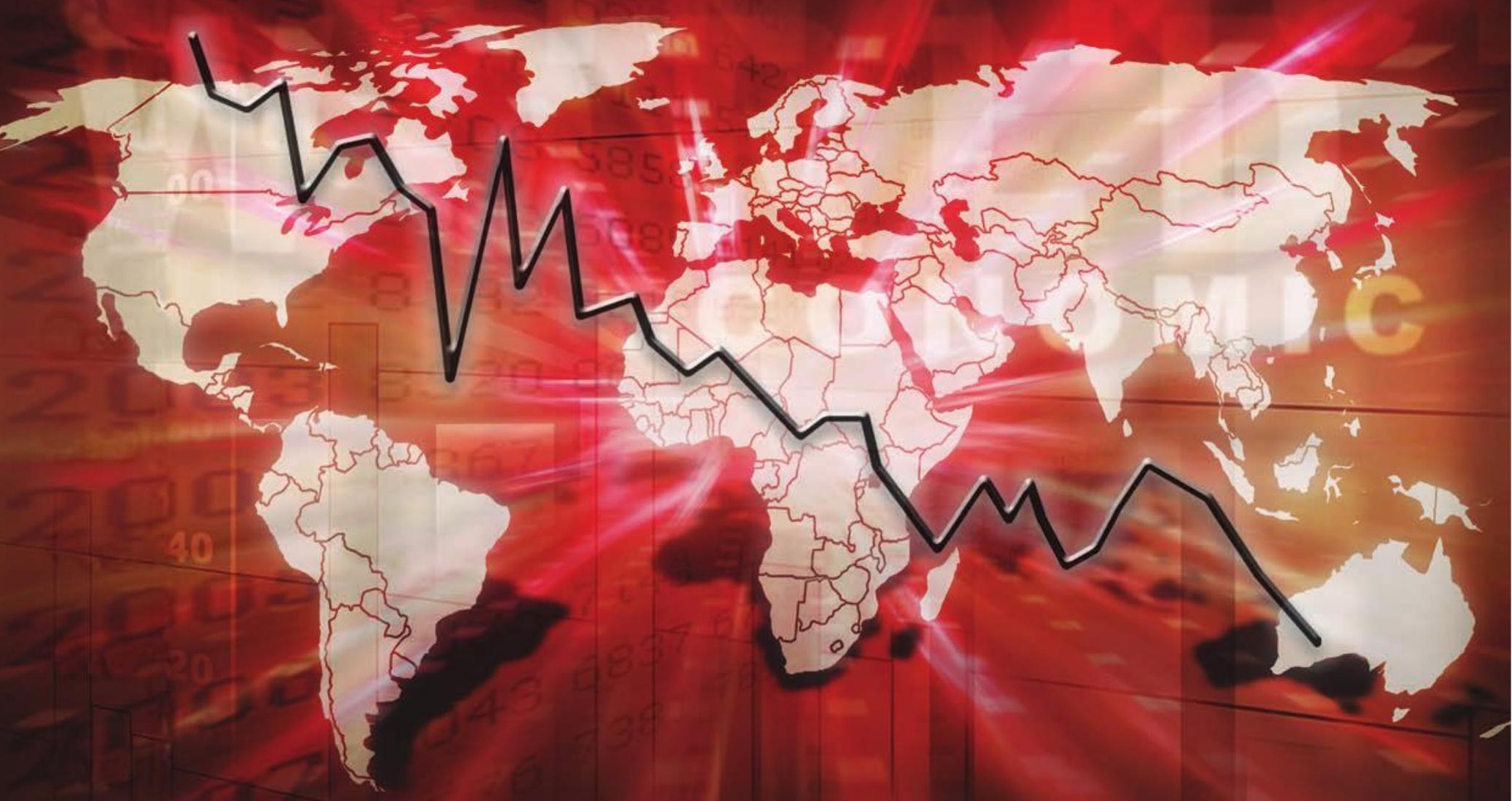

Martin Kaae Jensen, University of Leicester

Working Paper No. 15/16

July 2015 


\title{
Existence, Uniqueness, and Comparative Statics in Contests
}

\author{
Martin Kaae Jensen*
}

July 23, 2015

\begin{abstract}
Many important games are aggregative allowing for robust comparative statics analysis even when a game does not exhibit strategic complements or substitutes (Acemoglu and Jensen (2013)). This paper establishes such comparative statics results for contests improving upon existing results by (i) allowing payoff functions to be discontinuous at the origin, and (ii) allowing for asymmetric rent-seeking contests and patent races. A leading example where (i) is relevant is the classical Tullock contest (Tullock (1980)). The paper also studies existence and uniqueness of equilibria extending the results of Szidarovszky and Okuguchi (1997) and Cornes and Hartley (2005) to patent races.
\end{abstract}

Keywords: Comparative statics, Uniqueness, Existence, Contest, Rent-seeking, Aggregative game, Local solvability condition.

JEL Classification Codes: C61, D80, D90, E20, I30.

*Department of Economics, University of Leicester. (e-mail: mj182@le.ac.uk) 


\section{Introduction}

In contests, agents exert effort to increase their chance of winning a monetary prize, capturing a patent, or defeating their enemies. The study of contests dates back to Loury (1979) and Tullock (1980) and forms a large and active research area. Contests have been used to analyze R\&D rivalry (patent races), various types of rent-seeking behavior, financial institutions, market share competition, conflicts, and a number of other strategic interactions. For a long list of applications and further references see Skaperdas (1996), Nti (1997), and Corchón (2007). For a recent contribution to the literature see Kolmar and Wagener (2013).

Szidarovszky and Okuguchi (1997) prove the existence of a unique pure strategy Nash equilibrium in the standard case where one of the contenders always wins the prize. Exploiting methods from the literature on aggregative games, Cornes and Hartley (2005) give a very simple proof of the same result. Nti (1997) derives comparative statics results for symmetric contests, allowing for the possibility that the agents win the prize with probability less than 1 . This possibility has merit since a contests where no agent necessarily wins is equivalent to a patent race (Loury (1979)). ${ }^{1}$ Acemoglu and Jensen (2013) provide comparative statics results that apply to asymmetric contests and asymmetric patent races but their results, at least when taken at face value, require payoff functions to be differentiable which rules out situations where the contest success function is not continuous at the origin. Just like Cornes and Hartley (2005), Acemoglu and Jensen (2013) explicitly exploit the aggregative games structure of contests and patent races. ${ }^{2}$

The existing literature leaves open two question both of which are addressed in this paper.

- Firstly, does the existence and uniqueness result of Szidarovszky and Okuguchi (1997) and Cornes and Hartley (2005) extend to situations where there is a positive probability that no agent wins the prize. In particular, does this result extend to patent races in the spirit of Loury (1979)?

- Secondly, can comparative statics results similar to those of Acemoglu and Jensen (2013) be established when contest success functions are discontinuous at the origin?

The answer is in the affirmative in both cases: the comparative statics results in Acemoglu and Jensen continue to hold when contest success functions are discontinuous; and the existence and uniqueness of standard contests continues to hold when there is a positive probability that no agent wins the prize (in particular, in patent races).

The structure of the paper is as follows: In Section 2 the model and basic assumptions are introduced and discussed. Section 3 derives backward reply functions and proves existence and uniqueness of equilibrium. Finally, Section 4 contains the comparative statics results.

\footnotetext{
${ }^{1}$ This relationship is further elaborated on in Section 2, see also Nti (1997).

${ }^{2}$ For a general discussion of aggregative games see Jensen (2010).
} 


\section{Models of Contests and Patent Races}

Consider a contest with $I \in \mathbb{N}$ agents fighting for a prize. Agent $i \in \mathscr{I}$ 's payoff function is,

$$
\pi_{i}\left(s_{i}, s_{-i}\right)=V_{i} \cdot \frac{h_{i}\left(s_{i}\right)}{R+\sum_{j=1}^{I} h_{j}\left(s_{j}\right)}-c_{i}\left(s_{i}\right),
$$

where $s_{i} \in S_{i}=\left[0, \bar{s}_{i}\right] \subseteq \mathbb{R}_{+}$denotes effort, $c_{i}: \mathbb{R}_{+} \rightarrow \mathbb{R}_{+}$is the agent's cost function, and $V_{i}>0$ his individual valuation of the prize. ${ }^{3}$ The functions $h_{1}, \ldots, h_{I}: \mathbb{R}_{+} \rightarrow \mathbb{R}_{+}$and the constant $R \geq 0$ determine the contest success functions $\frac{h_{i}\left(s_{i}\right)}{R+\sum_{j=1}^{I} h_{j}\left(s_{j}\right)}, i \in \mathscr{I}$, which map a given profile of efforts $s=\left(s_{1}, \ldots, s_{I}\right)$ into the agents' winning probabilities.

Two classical references are Tullock (1980) and Loury (1979). Loury considers the case of a symmetric patent race with $h_{i}\left(s_{i}\right)=h\left(s_{i}\right), h$ a concave and strictly increasing function, $c_{i}\left(s_{i}\right)=$ $R s_{i}$ where $R>0$ is the interest rate which also affects the agents' probability of winning the race (Loury (1979), p.399; see also the paragraph prior to Theorem 2 in this paper). Patent races may be viewed as contests where a non-strategic agency (the house in gambling terminology) has a certain probability of winning the prize. Indeed when $R>0$, the probability that no contender wins the prize is $1-\frac{\sum_{i} h_{i}\left(s_{i}\right)}{R+\sum_{i} h_{i}\left(s_{i}\right)}>0$ and this is then also the probability that the house wins. In contrast, contests a la Tullock have $R=0$ and $c_{i}\left(s_{i}\right)=s_{i}$. Tullock considers symmetric rent-seeking games with $h_{i}\left(s_{i}\right)=s_{i}^{k}, k \in(0,1], i=1,2$. The more general specification of contests with riskneutral agents has again $c_{i}\left(s_{i}\right)=s_{i}$, but allows $h_{i}\left(s_{i}\right)$ to be arbitrary concave and strictly increasing functions. ${ }^{4}$ For an axiomatization of various contest success functions including that of Tullock (1980) and the logit function of Hirshleifer (1989) returned to below, see Skaperdas (1996). Here we allow for all of the previous cases and their asymmetric extensions as long as the following standard assumption is satisfied:

Assumption 1 For all $i \in \mathscr{I}: h_{i}: \mathbb{R}_{+} \rightarrow \mathbb{R}_{+}$and $c_{i}: \mathbb{R}_{+} \rightarrow \mathbb{R}_{+}$are twice continuously differentiable and strictly increasing. Furthermore, zero effort implies zero likelihood of winning the prize, i.e., $h_{i}(0)=0$.

Note that under Assumption 1, payoffs are not well-defined when $R=0$ and $s=(0, \ldots, 0)$. The contest literature's convention is to set the probability of winning the price equal to $\frac{1}{I}$ in this situation, and so define $\pi_{i}(0)=V_{i} \frac{1}{I}-c_{i}(0)$ when $R=0$. If all agents $j \in \mathscr{I} \backslash\{I\}$ choose $s_{j}=0$, it is clear from the fact that $c_{i}$ is continuous that it is not optimal for agent $i$ to also choose $s_{i}=0$. Hence $s=(0, \ldots, 0)$ cannot be a Nash equilibrium under Assumption 1 when $R=0$. In order to also rule out the trivial equilibrium $s=(0, \ldots, 0)$ when $R>0$, we impose the following boundary condition: ${ }^{5}$

\footnotetext{
${ }^{3}$ Note that strategy sets are assumed to be compact. This is for technical convenience. If one insists on having $S_{i}=\mathbb{R}_{+}$, compactness of strategy sets can instead be derived as a condition that must necessarily hold in equilibrium by assuming, for example, that for all $s_{-i} \in \mathbb{R}_{+}^{I-1}, \pi_{i}\left(\cdot, s_{-i}\right)$ strictly decreases for $s_{i} \geq \bar{s}_{i}$.

${ }^{4}$ Note that unit marginal costs $c_{i}\left(s_{i}\right)=s_{i}$ results when agents are risk-neutral, win the prize with probabilities $p_{i}(s) \equiv$ $\frac{h_{i}\left(s_{i}\right)}{\sum_{j=1}^{I} h_{j}\left(s_{j}\right)}, i \in \mathscr{I}$, and the status quo has value $I_{i}$. Indeed, an agent's expected payoff is then $p_{i}(s)\left[V_{i}+I_{i}-s_{i}\right]+(1-$ $\left.p_{i}(s)\right)\left[I_{i}-s_{i}\right]=p_{i}(s) V_{i}+I_{i}-s_{i}$ which is (1) with $c_{i}\left(s_{i}\right)=s_{i}$ once the strategically irrelevant term $I_{i}$ has been removed.

${ }^{5}$ Note that Assumption 2 is implied by Assumption 1 when $R=0$ (specifically, from the fact that $h_{i}$ is strictly increasing).
} 
Assumption 2 For all $i \in \mathscr{I}, V_{i} h_{i}^{\prime}(0)>R c_{i}^{\prime}(0)$.

To see that $s=(0, \ldots, 0)$ cannot be an equilibrium when $R>0$ and Assumption 2 holds, consider the marginal payoff of agent $i$ :

$$
D_{s_{i}} \pi_{i}\left(s_{i}, s_{-i}\right)=V_{i} h_{i}^{\prime}\left(s_{i}\right) \cdot \frac{R+\sum_{j=1}^{I} h_{j}\left(s_{j}\right)-h_{i}\left(s_{i}\right)}{\left(R+\sum_{j=1}^{I} h_{j}\left(s_{j}\right)\right)^{2}}-c_{i}^{\prime}\left(s_{i}\right) .
$$

Thus $D_{s_{i}} \pi_{i}(0, \ldots, 0)=V_{i} h_{i}^{\prime}(0) \frac{1}{R}-c_{i}^{\prime}(0)$ which under Assumption 2 is strictly positive. Hence it is not optimal for agent $i$ to choose $s_{i}=0$ when everyone else also chooses to exert zero effort.

The key assumption for our results imposes the curvature condition of Acemoglu and Jensen (2013),

Assumption 3 For all $i \in \mathscr{I}$ :

$$
\frac{h_{i}^{\prime \prime}\left(s_{i}\right)}{h_{i}^{\prime}\left(s_{i}\right)} \leq \frac{c_{i}^{\prime \prime}\left(s_{i}\right)}{c_{i}^{\prime}\left(s_{i}\right)} \text { for all } s_{i} \in S_{i} .
$$

Note that if $c_{i}$ is linear, Assumption 3 says that $h_{i}$ must be concave. If $h_{i}$ is linear, Assumption 3 requires $c_{i}$ to be convex. In the setting of Tullock (1980) mentioned above we have,

$$
\pi_{i}\left(s_{i}, s_{-i}\right)=V \cdot \frac{s_{i}^{k}}{\sum_{j=1}^{I} s_{j}^{k}}-s_{i},
$$

Hence (3) holds since $k \leq 1$. In logit contests,

$$
\pi_{i}\left(s_{i}, s_{-i}\right)=V \cdot \frac{h\left(s_{i}\right)}{\sum_{j=1}^{I} h\left(s_{j}\right)}-s_{i},
$$

(3) holds because $h$ is concave. But in general, concavity of $h_{i}$ and convexity of $c_{i}$ are not necessary for (3) to be satisfied. Hirshleifer (1989) proposes taking $h_{i}\left(s_{i}\right)=e^{k s_{i}}(k>0)$ in the logit case which defines a convex function $h_{i}$. In this situation, (3) will be satisfied if, for example, $c_{i}\left(s_{i}\right)=e^{l s_{i}}$ where $l \geq k$.

\section{Backward Replies, Existence and Uniqueness}

The analysis in this paper is based on backward reply functions (Selten (1970), Novshek (1985), Kukushkin (1994), Acemoglu and Jensen (2013)). For a strategy profile $s$, define the aggregate as $Q=\sum_{i} h_{i}\left(s_{i}\right)$. Given this definition, we may write the marginal payoff of agent $i$ (see (2)) as a function of $Q$ and the agent's own strategy $s_{i}$ :

$$
\Psi_{i}\left(s_{i}, Q\right) \equiv V_{i} h_{i}^{\prime}\left(s_{i}\right) \cdot \frac{R+Q-h_{i}\left(s_{i}\right)}{(R+Q)^{2}}-c_{i}^{\prime}\left(s_{i}\right),
$$

When the payoff function is pseudo-concave in $s_{i}$, it is clear that $s_{i}$ is an interior optimizer for agent $i$ if and only if $\Psi_{i}\left(s_{i}, Q\right)=0$ and $Q=\sum_{j} h_{j}\left(s_{j}\right)$. We may, however, also think of $Q \in \mathbb{R}$ as an 
independent variable and given $Q$ look for solutions to $\Psi_{i}\left(s_{i}, Q\right)=0$. Any such solution is called a backward reply.

Consider now the following definition from Acemoglu and Jensen (2013):

Definition 1 (Uniform Local Solvability) Agent $i \in \mathscr{I}$ satisfies the uniform local solvability condition if $\Psi_{i}\left(s_{i}, Q\right)=0 \Rightarrow D_{s_{i}} \Psi_{i}\left(s_{i}, Q\right)<0$ for all $s_{i} \in S_{i}$, and $Q>0$.

As shown under slightly more general conditions in Acemoglu and Jensen (2013), the payoff functions in (1) satisfy the uniform local solvability condition when Assumption 3 holds. To see why, calculate,

$$
D_{s_{i}} \Psi_{i}\left(s_{i}, Q\right)=V_{i} h_{i}^{\prime \prime}\left(s_{i}\right) \cdot \frac{R+Q-h_{i}\left(s_{i}\right)}{(R+Q)^{2}}-c_{i}^{\prime \prime}\left(s_{i}\right)-V_{i} \frac{\left(h_{i}^{\prime}\left(s_{i}\right)\right)^{2}}{(R+Q)^{2}}
$$

When $\Psi_{i}\left(s_{i}, Q\right)=0$, this derivative reduces to,

$$
D_{s_{i}} \Psi_{i}=\frac{h_{i}^{\prime \prime}\left(s_{i}\right)}{h_{i}^{\prime}\left(s_{i}\right)} c_{i}^{\prime}\left(s_{i}\right)-c_{i}^{\prime \prime}\left(s_{i}\right)-V_{i} \cdot \frac{\left(h_{i}^{\prime}\left(s_{i}\right)\right)^{2}}{(R+Q)^{2}} .
$$

Dividing both sides by $c_{i}^{\prime}\left(s_{i}\right)>0$, we immediately see $D_{s_{i}} \Psi_{i}<0$ when Assumption 3 holds.

That $D_{s_{i}} \Psi_{i}\left(s_{i}, Q\right)<0$ for all $s_{i}$ and $Q$ implies that when we fix $Q$, the function $\Psi_{i}\left(s_{i}, Q\right)$ is strictly decreasing in $s_{i}$ at any zero, i.e., at any $s_{i}$ with $\Psi_{i}\left(s_{i}, Q\right)=0$. Since it is also continuous, it follows that there exists at most one backward reply for any given $Q>0$. We may therefore define the backward reply function,

$$
s_{i}=b_{i}(Q) \Leftrightarrow \Psi_{i}\left(s_{i}, Q\right)=0 .
$$

Given the individual backward reply functions $\left(b_{i}\right)_{i \in \mathscr{I}}$ we can then define the aggregate backward reply function:

$$
z(Q)=\sum_{i} h_{i}\left(b_{i}(Q)\right)
$$

Note that $z$ will be continuous when $Q>0$ (this follow from the implicit function theorem). Note also that the current treatment ignores both the problems related to possible boundary solutions and the question of whether in fact $z$ is well-defined on an appropriate domain. Both are serious concerns. But both are treated in detail in Acemoglu and Jensen (2013), in particular it is shown there that we do not need to worry about boundary solutions under the uniform local solvability condition.

Definition 2 An equilibrium aggregate is a fixed point of the aggregate backward reply function, i.e., $a Q^{*} \geq 0$ such that

$$
z\left(Q^{*}\right)=Q^{*}
$$

If $Q^{*}$ is an equilibrium aggregate, then $\left(b_{i}\left(Q^{*}\right)\right)_{i \in \mathscr{I}}$ is a Nash equilibrium for the original game. Conversely, if $\left(s_{i}^{*}\right)_{i \in \mathscr{I}}$ is a Nash equilibrium, then $\sum_{i} h_{i}\left(s_{i}^{*}\right)$ is an equilibrium aggregate. Thus there is a one-to-one correspondence between equilibrium aggregates and Nash equilibria of the contest. 
Lemma 1 Let Assumptions 1-3 be satisfied. Then there exist $Q_{H}>Q_{L}>0$ such that

$$
z(Q)>Q \text { for all } Q \in\left(0, Q_{L}\right) \text { and } z(Q)<Q \text { for all } Q>Q_{H}
$$

Proof. The existence of $Q_{H}$ follows immediately from the compactness of the strategy sets. Also, when $R>0$ the existence of $Q_{L}$ follows immediately from the fact that $z(0)$ is well defined, $z$ is continuous continuous, and $b_{i}(0)>0$ for all $i$ by Assumption 2. In the following we therefore only need to establish the existence of $Q_{L}$ in the case where $R=0$. $\tilde{b}_{i}$ by,

Consider $Q>0$ and let $\tilde{s}_{i}=\frac{h_{i}\left(s_{i}\right)}{Q}$. Since $h_{i}$ is strictly increasing by Assumption 1, we may define

$$
\tilde{s}_{i}=\tilde{b}_{i}(Q) \Leftrightarrow V_{i} \frac{Q\left(1-\tilde{s}_{i}\right)}{Q^{2}}-\frac{c_{i}^{\prime}\left(h_{i}^{-1}\left(Q \tilde{s}_{i}\right)\right)}{h_{i}^{\prime}\left(h_{i}^{-1}\left(Q \tilde{s}_{i}\right)\right)}=0 .
$$

If there exists $Q_{L}>0$ such that $\tilde{b}_{i}(Q) \geq 1$ for all $Q<Q_{L}$ we are clearly done since then $z(Q)=$ $\sum_{i} h_{i}\left(b_{i}(Q)\right) \geq I Q>Q$ whenever $Q>0$. So consider from now on the case where this is not so. There will then exist a sequence $\left(Q^{n}\right)$ with $Q^{n} \downarrow 0$ so that for all $n, \tilde{b}_{i}\left(Q^{n}\right)<1$. Consider a convergent subsequence $\lim _{m \rightarrow \infty} \tilde{b}_{i}\left(Q^{n_{m}}\right)=\hat{s}_{i}$. Evidently $\hat{s}_{i} \leq 1$. Under Assumptions $1-3, \frac{c_{i}^{\prime}\left(h_{i}^{-1}(z)\right)}{h_{i}^{\prime}\left(h_{i}^{-1}(z)\right)}$ is increasing in $z$ and therefore $\lim _{z \downarrow 0} \frac{c_{i}^{\prime}\left(h_{i}^{-1}\left(Q \tilde{S}_{i}\right)\right)}{h_{i}^{\prime}\left(h_{i}^{-1}\left(Q \tilde{s}_{i}\right)\right)}<+\infty$. Hence we must have $\hat{s}_{i}=1$ in order for (9) not to be violates for some $m$. So in fact $\tilde{b}_{i}\left(Q^{n_{m}}\right) \uparrow 1$. Since this applies to the limit point of any convergent subsequence, we have $\tilde{b}_{i}(Q) \uparrow 1$ as $Q \downarrow 0$ (in particular, $\tilde{b}_{i}(\cdot)$ is continuous from above when we set $\left.\tilde{b}_{i}(0)=1\right)$. Hence for any $\epsilon>0$ we can find a $Q_{L}$ so that $Q<Q_{L} \Rightarrow \tilde{b}_{i}(Q) \geq 1-\epsilon$. Equivalently $h_{i}\left(b_{i}(Q)\right) \geq Q-\epsilon Q$ and so $z(Q)=\sum_{i} h_{i}\left(b_{i}(Q)\right) \geq I Q-\epsilon I Q=(I-\epsilon) Q>Q$.

Theorem 1 Let Assumptions 1-3 be satisfied. Then there exists a unique pure strategy Nash equilibrium $s^{*}=\left(s_{1}^{*}, \ldots, s_{I}^{*}\right)$, and this equilibrium is non-trivial, i.e., $s^{*} \neq(0, \ldots, 0)$.

Proof. Existence follows from Lemma 1 and the mean value theorem. That $s^{*} \neq(0, \ldots, 0)$ whether the equilibrium $s^{*}$ is unique or not was established in-text in the paragraphs before and after equation (2). To prove uniqueness, we exploit the share function of Cornes and Hartley (2005). Let $\tilde{s}_{i}=\frac{h_{i}\left(s_{i}\right)}{Q}$, substitute into (6) and set equal to zero:

$$
V_{i} \cdot \frac{R+Q-Q \tilde{s}_{i}}{(R+Q)^{2}}-\frac{c_{i}^{\prime}\left(h_{i}^{-1}\left(Q \tilde{s}_{i}\right)\right)}{h_{i}^{\prime}\left(h_{i}^{-1}\left(Q \tilde{s}_{i}\right)\right)}=0
$$

Write $\tilde{s}_{i}=\tilde{b}_{i}(Q)$ if and only if the previous equation is satisfied. Our objective is to show for all $i, \tilde{b}_{i}$ is non-increasing in $Q$ in the neighborhood of any equilibrium aggregate $Q=Q^{*}$, while for at least one $i, \tilde{b}_{i}$ must in be strictly decreasing in $Q$ at $Q^{*}$. Since it then follows that $\sum_{j} \tilde{b}_{j}(Q)$ is strictly decreasing at any equilibrium aggregate $Q^{*}$, i.e., when $\sum_{j} \tilde{b}_{j}\left(Q^{*}\right)=1$, there can be at most one equilibrium aggregate. Equivalently, there is at most one $Q^{*}$ such that $z\left(Q^{*}\right)=\sum_{j} b_{i}\left(Q^{*}\right)=$ $Q^{*}$. Since $Q^{*}$ uniquely determines the equilibrium efforts $s_{i}^{*}=b_{i}\left(Q^{*}\right), i=1, \ldots, I$, this implies the conclusion of the Theorem. 
That $\tilde{b}_{i}$ is non-increasing implies that if $Q$ and $\tilde{s}_{i}$ satisfy (10), then we cannot increase $Q$ and weakly increase $\tilde{s}_{i}$ and still satisfy (10). To see that this is indeed true, note first that $\frac{c_{i}^{\prime}\left(h_{i}^{-1}\left(Q \tilde{s}_{i}\right)\right)}{h_{i}^{\prime}\left(h_{i}^{-1}\left(Q \tilde{s}_{i}\right)\right)}$ is non-decreasing in $Q \tilde{s}_{i}$ under Assumption 3 and therefore weakly increases when $Q$ and/or $\tilde{s}_{i}$ are increased. Next consider

$$
\frac{R+Q-Q \tilde{s}_{i}}{(R+Q)^{2}}=\frac{R+Q\left(1-\tilde{s}_{i}\right)}{(R+Q)^{2}}
$$

Clearly, this term is strictly decreasing in $\tilde{s}_{i}$ (here and below we need only consider $\tilde{s}_{i} \leq 1$ and $Q>0$ where the latter is because $Q=0$ cannot be an equilibrium aggregate). To see that (11) is non-increasing in $Q$ under the condition of the Theorem differentiate w.r.t. $Q$ :

$$
\frac{\left(1-\tilde{s}_{i}\right)(R+Q)^{2}-2(R+Q)\left(R+Q\left(1-\tilde{s}_{i}\right)\right)}{(R+Q)^{4}}
$$

So (11) is non-increasing in $Q$ if and only if:

$$
\begin{gathered}
\left(1-\tilde{s}_{i}\right)(R+Q)^{2}-2(R+Q)\left(R+Q\left(1-\tilde{s}_{i}\right)\right) \leq 0 \Leftrightarrow\left(1-\tilde{s}_{i}\right)(R+Q)-2\left(R+Q\left(1-\tilde{s}_{i}\right)\right) \leq 0 \Leftrightarrow \\
\left(1-\tilde{s}_{i}\right)(R-Q)-2 R \leq 0
\end{gathered}
$$

This inequality clearly holds if $R=0$. If $R>0$ there are two cases. Either $Q \geq R$, in which case $\left(1-\tilde{s}_{i}\right)(R-Q)-2 R \leq-2 R<0$. If $Q<R,\left(1-\tilde{s}_{i}\right)(R-Q)-2 R<(R-Q)-2 R=-R-Q<0$. So in all cases, $\tilde{b}_{i}(Q)$ is non-increasing when $Q$ is an equilibrium aggregate. In fact, since for at least one agent we must have $\tilde{s}_{i}<1$ in equilibrium, the previous inequalities must be strict for at least one agent $i$ which implies that $\tilde{b}_{i}$ is strictly decreasing for that agent. This finishes the proof.

As discussed in the Introduction, Szidarovszky and Okuguchi (1997) and Cornes and Hartley (2005) establish all of the conclusion of Theorem 1 in the case where $R=0$. The proof of uniqueness in Theorem 1 follows the basic strategy of Cornes and Hartley (2005) in using share functions which are very effective in dealing with uniqueness issues in aggregative games.

\section{Comparative Statics}

Contests and patent races are neither games of strategic complements or strategic substitutes. They are, however, aggregative which provides enough structure to develop robust comparative statics results. ${ }^{6}$ The most general results available in the literature for the class of games considered in this paper are due to Nti (1997) and Acemoglu and Jensen (2013). Nti's results apply to symmetric equilibria in symmetric games (in particular, agents must be identical) where $c_{i}\left(s_{i}\right)=s_{i}$ for all $i$. Acemoglu and Jensen (2013) provide results for asymmetric contests and rent seeking games, but their setting requires payoff functions to be everywhere differentiable. As discussed in Section 2 , this is violated when $R=0$ at the point $s=(0, \ldots, 0)$. The Theorem that follows next can be viewed as an improved version of Proposition 3 in Acemoglu and Jensen (2013) which allow for the discontinuous payoffs of many contests. The critical "fix" is in this connection Lemma 1.

\footnotetext{
${ }^{6}$ For the state-of-the-art in games that are not aggregative and also do not exhibit strategic complements see Roy and Sabarwal (2010) and Monaco and Sabarwal (2015).
} 
Definition 3 There is a positive shock to agent $i \in \mathscr{I}$, if the agent's payoff function $\pi_{i}(s)=V_{i} \frac{h_{i}\left(s_{i}\right)}{R+\sum_{j} h_{j}\left(s_{j}\right)}-$ $c_{i}\left(s_{i}\right)$ changes to $\tilde{\pi}_{i}(s)=\tilde{V}_{i} \frac{\tilde{h}_{i}\left(s_{i}\right)}{\tilde{R}+\sum_{j} \tilde{h}_{j}\left(s_{j}\right)}-\tilde{c}_{i}\left(s_{i}\right)$ where $D_{s_{i}} \tilde{\pi}_{i}(s) \geq D_{s_{i}} \pi_{i}(s)$ for all $s \in S$.

A simple example of a positive shock to agent $i$ is an increase in his valuation of the price from $V_{i}$ to $\tilde{V}_{i}$. Indeed, one clearly has

$$
\tilde{V}_{i} \frac{\partial \frac{h_{i}\left(s_{i}\right)}{R+\sum_{j} h_{j}\left(s_{j}\right)}}{\partial s_{i}}-c_{i}^{\prime}\left(s_{i}\right) \geq V_{i} \frac{\partial \frac{h_{i}\left(s_{i}\right)}{R+\sum_{j} h_{j}\left(s_{j}\right)}}{\partial s_{i}}-c_{i}^{\prime}\left(s_{i}\right)
$$

If $c_{i}$ is replaced with $\tilde{c}_{i}$ where $\tilde{c}_{i}^{\prime}\left(s_{i}\right) \leq c_{i}^{\prime}\left(s_{i}\right)$, i.e., if the agent experiences a pointwise decrease in marginal costs, then this is also a positive shock to agent $i$. A decrease in $R$ will be a positive shock for all agents $i \in \mathscr{I}$ both when the cost function is held fixed and when $R$ enters the costs function as in Loury (1979), $c_{i}\left(s_{i}\right)=R s_{i}{ }^{7}$ It is also possible to consider changes in the functions $h_{i}$ but the issue will not be pursued here.

Theorem 2 Consider a contest with payoff functions (1) and suppose that Assumptions 1-3 are satisfied. Then:

1. The equilibrium aggregate is increasing in any positive shock to one or more of the agents (e.g., a decrease in $R$, an increase in $V_{i}$ for one or more agents, or a pointwise decrease in marginal $\operatorname{costs} c_{i}^{\prime}\left(s_{i}\right)$ for one of more agents).

2. Entry of an additional agent increases the equilibrium aggregate.

3. If at the corresponding equilibrium $s^{*}$ with aggregate $Q^{*}=\sum_{i} h_{i}\left(s_{i}^{*}\right)$, agent $i$ is an absolute favorite to win in the sense that,

$$
\frac{h_{i}\left(s_{i}^{*}\right)}{\left(R+\sum_{i} h_{i}\left(s_{i}^{*}\right)\right)} \geq \frac{1}{2}
$$

then agent $i$ 's equilibrium effort increases in any of the situations of 1 and 2. Conversely, if,

$$
\frac{h_{i}\left(s_{i}^{*}\right)}{\left(R+\sum_{i} h_{i}\left(s_{i}^{*}\right)\right)}<\frac{1}{2}
$$

then the changes in parts 1 and 2 decrease agent i's effort provided that the shock does not affect this agent directly (e.g., corresponding to a decrease in another agent's marginal costs, or entry of an additional agent).

Proof. Taking Lemma 1 into account, both 1 and 2 are direct consequences of the results in Acemoglu and Jensen (2013) because we may restrict $Q$ to an interval $\left[Q_{L}-\epsilon, Q_{H}+\epsilon\right], \epsilon>0$ and $Q_{L}-\epsilon>0$ where the discontinuity at the origin is irrelevant. The proofs will not be reproduced

\footnotetext{
${ }^{7}$ Note that the payoff of an agent in Loury is in fact $\frac{V}{R} \frac{h_{i}\left(s_{i}\right)}{R+\sum_{j} h_{j}\left(s_{j}\right)}-s_{i}$ (see equation (7) on page 399 of Loury (1979)). Multiplying with $R$ - which does not alter the optimal decision - leads to the functional form considered here.
} 
here. 3. Consider first the case where the shock does not affect agent $i$. We already know that $Q$ increases in equilibrium. Applying the implicit function theorem to $\Psi_{i}\left(s_{i}, Q\right)=0$ at an equilibrium, we get that $\frac{d s_{i}}{d Q}=-\frac{D_{Q} \Psi_{i}\left(s_{i}^{*}, Q^{*}\right)}{D_{s_{i}} \Psi_{i}\left(s_{i}^{*}, Q^{*}\right)}$. Since $D_{s_{i}} \Psi_{i}\left(s_{i}^{*}, Q^{*}\right)<0$ by the uniform local solvability condition and $Q$ is increasing in the positive shock, the first claim follows immediately when $D_{Q} \Psi_{i}\left(s_{i}^{*}, Q^{*}\right) \geq 0$. Since,

$$
D_{Q} \Psi_{i}\left(s_{i}, Q\right) \equiv V_{i} h_{i}^{\prime}\left(s_{i}\right) \cdot\left[-(R+Q)^{-2}+2 h_{i}\left(s_{i}\right)(R+Q)^{-3}\right]
$$

It is seen that $D_{Q} \Psi_{i} \geq 0$ if and only if:

$$
2 h_{i}\left(s_{i}\right)(R+Q)^{-3} \geq(R+Q)^{-2} \Leftrightarrow h_{i}\left(s_{i}\right) \geq \frac{1}{2}(R+Q)
$$

which in turn is equivalent to the condition of the theorem.

When $D_{Q} \Psi_{i}<0$, the increase in $Q^{*}$ will lead to a decrease in agent $i$ 's strategy by the exact same argument.

If the shock affects agent $i$, it will in the first of the two cases lead to a further increase in $s_{i}$ and so the conclusion that $s_{i}$ increases remains the same. In the second case, this is not so and it is therefore necessary to assume that the shock does not affect agent $i$ directly.

Note that when $R=0$, condition (12) simply says that the agent will win the contest with at least $50 \%$ probability. In the two-agent setting, Dixit (1987) refers to such a agent as a favorite. When $R>0$, the interpretation remains the same in two-agent contests with a house that wins with probability $\frac{R}{R+\sum_{i} h_{i}\left(s_{i}^{*}\right)}$. With more than two agents, (12) says that the agent must be the favorite to win when pitched again everyone else including the house. This is what is meant by the term absolute favorite. So what 3 of Theorem 2 tells us is that an absolute favorite will actively defend her position by increasing her effort given any change in the game that leads to an overall increase in effort (and hence would lead to a decrease in her probability of winning if her effort remained the same). In particular, she increases her effort if another agent enters the game or another agent experiences a decrease in marginal costs, or another agent's valuation of the prize increases. Conversely, a agent who is not more likely to win than everybody else combined is "cowed" and lowers her effort and probability of winning the prize when, for example, another agent's marginal costs decrease or another agent's valuation of the prize increases.

\section{Conclusion}

This paper contributes to the literature on contests by establishing existence, uniqueness, and comparative statics results for asymmetric contests and patent races. The results exploit the fact that contests are aggregative games, and mathematically the proofs follow closely Cornes and Hartley (2005) and Acemoglu and Jensen (2013).

Acemoglu and Jensen (2013) establish comparative statics results for aggregative games that either exhibit strategic substitutes or satisfy a local solvability condition (see Definitions 7-8 in Acemoglu and Jensen (2013)). Contests are not games of strategic substitutes but they do satisfy the 
uniform local solvability condition (Definition 1 in the current paper or Definition 8 in Acemoglu and Jensen (2013)). While Acemoglu and Jensen assume that payoff functions are differentiable, the current results allow contest success functions to be discontinuous at the origin. As is clear from this paper's proofs, however, the extension is straight-forward. There is no doubt that one can similarly extend Acemoglu and Jensen (2013) to other situations where payoff functions are not everywhere differentiable (such as Cournot oligopolies with discontinuities at the origin, see von Mouche and Quartieri (2012)). One could also use the weaker version of the local solvability condition in Section 4.1.1 of Acemoglu and Jensen (2013) to derive comparative statics results for contests under weaker conditions than the curvature condition imposed here (Assumption 3). In particular, one can dispense with any differentiability conditions.

As for the existence and uniqueness results, the current paper's extension of Cornes and Hartley (2005) is again fairly straight-forward. Further extensions of the uniqueness part are likely to meet with limited success however. In fact, it is doubtful whether one can even establish uniqueness under reasonable conditions when contests are defined with general aggregators as in Acemoglu and Jensen (2013), Section 5.2.

\section{References}

Acemoglu, D. and M.K. Jensen (2013): “Aggregate Comparative Statics", Games and Economic Behavior 81, 27-49.

Corchón, L. (2007): “The Theory of Contests: a Survey”, Review of Economic Design 11, 69-100.

Cornes, R. and R. Hartley (2005): “Asymmetric Contests with General Technologies”, Economic Theory 26, 923-946.

Dixit, A. (1987): “Strategic Behavior in Contests”, American Economic Review 77, 891-898.

Hirshleifer, J. (1989): “Conflict and Rent-Seeking Success Functions: Ratio vs. Difference Models of Relative Success”, Public Choice 63, 101-112.

Jensen, M.K. (2010): “Aggregative Games and Best-reply Potentials”, Economic Theory 43, 45-66.

Kolmar, M. and A. Wagener (2013): "Inefficiency as a Strategic Device in Group Contests Against Dominant Opponents”, Economic Inquiry 51, 2083-2095.

Kukushkin, N.S. (1994): “A Fixed-point Theorem for Decreasing Mappings”, Economics Letters 46, 23-26.

Loury, G.C. (1979): “Market Structure and Innovation”, Quarterly Journal of Economics 93, 395-410.

A.J. Monaco and T. Sabarwal (2015): "Games with Strategic Complements and Substitutes", Economic Theory, forthcoming. 
Novshek, W. (1985): “On the Existence of Cournot Equilibrium”, Review of Economic Studies 52, 85-98.

Nti, K.O. (1997): “Comparative Statics of Contests and Rent-Seeking Games”, International Economic Review 38, 43-59.

Roy, S. and T. Sabarwal (2010): "Monotone Comparative Statics for Games with strategic Substitutes”, Journal of Mathematical Economics 46, 793-806.

Szidarovszky, F. and K. Okuguchi (1997): "On the Existence and Uniqueness of Pure Nash Equilibrium in Rent-Seeking Games", Games and Economic Behavior 18, 135-140.

Selten, R., Preispolitik der Mehrproduktenunternehmung in der Statischen Theorie, Springer Verlag, Berlin, 1970.

Skaperdas, S. (1992): “Cooperation, Conflict and Power in the Absence of Property Rights”, American Economic Review 82, 720-749.

Skaperdas, S. (1996): “Contest Success Functions”, Economic Theory 7, 283-290.

Tullock, G. (1980): “Efficient rent-seeking”. In: Buchanan, J.M., Tollison, R.D., Tullock, G. (Eds.), Toward a Theory of the Rent-Seeking Society. Texas A\&M Press, College Station, pp. 97-112.

von Mouche, P.H.M. and F. Quartieri (2012): "Existence of Equilibria in Cournotian Games with Utility Functions that are Discontinuous at the Origin", Working Paper, Available at http:// ssrn. com/abstract $=2528435$. 